\title{
Evains Wêche, Les Brasseurs de la ville
}

\section{Emanuela Cacchioli}

\section{(2) OpenEdition}

\section{Journals}

\section{Edizione digitale}

URL: http://journals.openedition.org/studifrancesi/5465

DOI: $10.4000 /$ studifrancesi.5465

ISSN: 2421-5856

\section{Editore}

Rosenberg \& Sellier

\section{Edizione cartacea}

Data di pubblicazione: 1 dicembre 2016

Paginazione: 579-580

ISSN: 0039-2944

\section{Notizia bibliografica digitale}

Emanuela Cacchioli, «Evains Wêche, Les Brasseurs de la ville », Studi Francesi [Online], 180 (LX | III) |

2016, online dal 01 janvier 2017, consultato il 18 septembre 2020. URL : http://

journals.openedition.org/studifrancesi/5465; DOI : https://doi.org/10.4000/studifrancesi.5465

\section{Questo documento è stato generato automaticamente il 18 settembre 2020.}

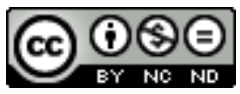

Studi Francesi è distribuita con Licenza Creative Commons Attribuzione - Non commerciale - Non opere derivate 4.0 Internazionale. 


\title{
Evains Wêche, Les Brasseurs de la ville
}

\author{
Emanuela Cacchioli
}

\section{NOTIZIA}

EVAINS WÊCHE, Les Brasseurs de la ville, Paris, Philippe Rey, 2016, 192 pp.

1 Opera prima di un giovane autore haitiano, Les Brasseurs de la ville è un romanzo ricco di spunti di riflessione che vede come protagonista una famiglia in estrema difficoltà economica trasferitasi nella capitale alla ricerca di condizioni di vita migliori. Sin dalle prime pagine, il lettore è proiettato a Cité Soleil e a Carrefour, due dei quartieri più poveri di Port-au-Prince e segue le vicende di un padre, una madre e cinque figli costretti ad affrontare le sfide della vita quotidiana per sopravvivere in un mondo dominato dalla povertà, dalla corruzione e dalle ingiustizie. I sette protagonisti del romanzo aspirano ad una rivincita sociale, a trasferirsi nei quartieri più eleganti della città, a raggiungere un riconoscimento pubblico e una discreta agiatezza. Per migliorare la loro esistenza, conducono una vita attiva e ingegnosa, convinti che prima o poi il destino riserverà loro piacevoli sorprese. La realtà è, tuttavia, ben diversa: l'illusione di avercela fatta, di aver finalmente ottenuto un avanzamento nella piramide sociale dura pochi istanti e le conquiste rivelano il loro lato più tragico e amaro. La lotta di questa famiglia è rappresentativa dell'esistenza di migliaia di haitiani che affrontano con coraggio e una certa dose di follia la vita quotidiana e sperano che il domani possa essere più roseo. Sono loro i «brasseurs», ossia coloro che si adattano a svolgere $\mathrm{i}$ mestieri più disparati pur di garantire un pezzo di pane ai propri figli. Ed è così che la madre si presenta ogni volta che le vengono chiesti chiarimenti sulla sua professione: "Je suis marchande ambulante de serviettes, parfois lessiveuse, parfois repasseuse. Je fais souvent la bonne à tout faire quand la rue ne donne rien. Cela dépend de la saison, vous voyez ce que je veux dire? Je suis une débrouilleuse» (p. 53). Se i genitori fanno il possibile per garantire la sopravvivenza della famiglia, anche i figli devono imparare a fare la loro parte. Proprio per questo le speranze dei genitori risiedono nei figli e l'avvenire della famiglia è rappresentato da Babette, la secondogenita, una ragazza avvenente e intelligente che sfrutta a suo vantaggio la relazione con un uomo ricco e 
molto più vecchio di lei per ottenere la vita lussuosa tanto agognata e permettere alla famiglia una maggiore agiatezza. Il misterioso signor Erikson sembra inizialmente un benefattore innamorato, salvo rivelare di tanto in tanto aspetti più spigolosi della personalità e strane relazioni molto potenti. E quando Babette scompare misteriosamente, il romanzo, che fino a quel momento aveva le tinte dell'epopea moderna, si trasforma in un racconto giallo, pieno di colpi di scena, di piste false e di soluzioni apparenti.

2 La famiglia al centro delle vicende è solo un pretesto per dare voce alla popolazione più povera e in difficoltà, a coloro che sono abitualmente esclusi e ignorati dalle classi sociali più abbienti. Questo aspetto risulta evidente dal fatto che, tranne Babette e i suoi fratelli, i personaggi non vengono quasi mai identificati dal loro nome proprio, ma soltanto dalla loro voce in prima persona che fornisce al lettore uno sguardo diretto e parziale della realtà e degli avvenimenti. La madre e il padre prendono la parola e si alternano nel racconto. Spiazzano il lettore perché non si annunciano e lasciano trapelare pochi indizi che permettano di identificare se si tratti del padre o della madre. I fatti vengono, dunque, ripresi e interpretati da diverse angolazioni e si intravvede lo scontro di mentalità tra l'uomo che è vittima del suo orgoglio e la donna che scende a compromessi pur di assicurare un futuro a se stessa e agli altri componenti della famiglia. Queste due voci rendono conto di una dimensione corale che emerge dalle pagine del romanzo e trova una sua rappresentazione nei pettegolezzi, vero passatempo del popolo che cerca di dimenticare la propria condizione di miseria. Si instaura una sorta di solidarietà che fa leva sulla condivisione di un vissuto comune, in cui è sempre possibile cogliere una prospettiva futura. La folla brulicante circonda la famiglia, la sostiene, la invidia o la compatisce. I singoli protagonisti hanno un loro status solo se vengono riconosciuti nella dimensione interpersonale e nelle dinamiche relazionali che si instaurano con gli altri. La Port-auPrince che emerge da queste pagine è una città fatta di colori, di profumi, del traffico asfissiante e di individui che affrontano con energia e un briciolo di follia i drammi quotidiani. Il romanzo vuole essere, inoltre, una critica al governo, alla corruzione che imperversa nel paese, alle ONG e alla comunità internazionale che promette fondi, alimenta false speranze e non interviene in modo efficace.

3 L'autore, Evains Wêche, è un giovane medico con una specializzazione in chirurgia ed esercita la professione di dentista. Nonostante la sua formazione, nutre da sempre un interesse per la letteratura e per la scrittura e organizza atelier culturali. Il suo talento viene scoperto da Gary Victor che lo incoraggia a coltivare la sua passione e lo coinvolge in una pubblicazione collettanea di racconti dal titolo Je ne savais pas que la vie serait si longue après la mort, uscita nel 2012 presso la casa editrice Mémoire d'encrier. L'anno successivo riceve il premio Henri Deschamps per la raccolta di novelle Le trou $d u$ voyeur. Nel 2014 esordisce con il suo primo romanzo che viene dato alle stampe da Mémoire d'encrier, ora ripubblicato in Francia da Philippe Rey.

4 Sentiremo ancora parlare di Evains Wêche e prepariamoci a leggere altri romanzi e racconti che affrontano questioni sociali, ideologiche ed economiche con un tono apparentemente leggero come avviene in Les Brasseurs de la ville, un romanzo in grado di rendere conto con estrema concretezza di una realtà multiforme, complessa e talvolta drammatica come quella haitiana. 